\title{
Efeito da Suplementação de L-Arginina Sobre a Secreção de Hormônio do Crescimento e Fator de Crescimento Semelhante à Insulina em Adultos
}

\begin{abstract}
RESUMO
Baseado nos pressupostos de que a infusão de aminoácidos pode aumentar a secreção de hormônio de crescimento $(\mathrm{GH})$, e que o metabolismo deste hormônio está relacionado com a secreção do fator de crescimento semelhante à insulina (IGF-I), o objetivo deste estudo foi verificar o efeito da suplementação de L-arginina sobre o GH e IGF-I em adultos. Participaram do estudo 17 indivíduos do sexo masculino, que foram randomizados para receber L-arginina $(n=10)$ ou placebo $(n=7)$, sete gramas ao dia, durante um período de sete dias. Antes e após o período de suplementação, os voluntários realizaram coleta de sangue em jejum para verificação dos níveis séricos de GH e IGF-I, bem como coleta de urina para verificação da excreção de uréia. Ao final do período experimental, verificamos que o grupo que recebeu L-arginina aumentou a excreção de uréia na urina (de 2684,1 \pm 475,2 mg/dl para 2967,2 \pm 409,7 $\mathrm{mg} / \mathrm{dl}, \mathrm{p}=0,002$ ), entretanto não modificou significativamente a secreção dos hormônios avaliados. O grupo que recebeu placebo não alterou significativamente nenhum parâmetro avaliado. A suplementação de Larginina durante sete dias mostrou-se ineficaz para aumentar a secreção de GH e IGF-I em indivíduos adultos do sexo masculino. (Arq Bras Endocrinol Metab 2007;51/4:587-592)
\end{abstract}

Descritores: L-arginina; Hormônio do crescimento; IGF-1; Suplementação

\section{ABSTRACT}

Effect of L-Arginine Supplementation on Secretion of Human Growth Hormone and Insuline-Like Growth Factor in Adults.

Based on presumptions that the infusion of amino acids can augment the release of human growth hormone $(\mathrm{hGH})$ and that this metabolism is related with secretion of insulin-like growth factor I (IGF-I), the purpose of this study was to verify the effect of L-arginine supplementation on $\mathrm{GH}$ and IGF-I in adults. Seventeen male individuals participated on the study and were randomized to receive $L$-arginine $(n=10)$ or placebo $(n=7)$, seven grams per day for seven days. Before and after the supplementation period, the volunteers realized blood collection in fasting to verify both $\mathrm{GH}$ and IGF-I levels, as well as urine collection to verify urea excretion. At the end of the experimental period, it was verified that the group that received Larginine augmented the urea in urine excretion (to $2684.1 \pm 475.2 \mathrm{mg} / \mathrm{dl}$ from $2967.2 \pm 409.7 \mathrm{mg} / \mathrm{dl}, \mathrm{p}=0.002$ ), therefore it did not alter significantly the release of hormones evaluated. The group which received placebo did not alter significantly any evaluated parameters. The L-arginine supplementation during seven days was ineffective to augment both GH and IGFI release in individual male adults. (Arq Bras Endocrinol Metab 2007;51/4:587-592)

Keywords: L-arginine; Human growth hormone; IGF-1; Supplementation artigo original

\author{
ana paula trussard Fayh \\ ROGÉRIO FRIEDMAN \\ KatIUCE BORgES SAPATA \\ Alvaro ReIschak de Oliveira
}

Laboratório de Pesquisa do Exercício (LAPEX) - Escola de Educação Física (APTF, KBS \& ARO) e Departamento de

Medicina Interna - Faculdade de Medicina (RF), Universidade Federal do Rio Grande do Sul (UFRGS), Porto Alegre, RS.

Recebido em 03/05/06 Revisado em 08/1 1/06 Aceito em 04/12/06 
$\mathrm{O}$ RIGINADO NOS SOMATOTROFOS hipofisários anteriores, o hormônio do crescimento (somatotropina, GH) é um potente agente anabólico. Ele promove o crescimento e a hipertrofia muscular pela facilitação do transporte de aminoácidos para o interior das células. Além disso, o GH estimula diretamente o metabolismo de gorduras (lipólise), aumentando a síntese de enzimas envolvidas nesse processo (1). No adulto, o GH facilita a síntese protéica aumentando o transporte de aminoácidos através das membranas plasmáticas, estimulando a formação de RNA, ou ativando os ribossomos celulares, que aumentam a síntese protéica (2).

A principal ação do $\mathrm{GH}$ sobre o crescimento é considerada indireta, uma vez que o hormônio do crescimento age diretamente sobre as células do fígado, ligando-se ao seu receptor e induzindo uma série de eventos que acabam resultando, por exemplo, na produção do fator de crescimento semelhante à insulina IGF-1 (ou somatomedina C) (3). Os IGFs são fatores de promoção do crescimento com estrutura molecular homóloga à insulina, encontrados na forma de IGF-1 e IGF-2. Estes fatores são sintetizados pelo fígado e pela maioria das células orgânicas (4). Os IGFs podem influenciar o crescimento, diferenciação e metabolismo celular e encontram-se ligados a proteínas carreadoras denominadas IGFBPs (5). O IGF-1 é um polipeptídeo produzido principalmente pelo fígado, e essa produção é dependente do $\mathrm{GH}$ (3).

A regulação da secreção de GH é complexa e pode sofrer diferentes influências. O hipotálamo interfere na síntese e liberação de GH através de dois neuropeptídeos: o hormônio liberador do GH - GHRH - e o hormônio inibidor da liberação de GH - GHRIH ou somatostatina. O IGF-1, cuja síntese é estimulada pelo GH, pode atuar sobre o hipotálamo, estimulando a secreção de somatostatina e, portanto, inibindo a liberação da síntese de GHRH, bem como sobre a hipófise, onde suprime a secreção e a expressão gênica do $\mathrm{GH}$ (6). Os fatores metabólicos que estimulam a liberação de $\mathrm{GH}$ são a hipoglicemia, a infusão ou a administração de aminoácidos, o exercício e o sono. A hipoglicemia é um dos mais potentes estímulos para a secreção do $\mathrm{GH}$, em função de uma citoglicopenia ocorre diminuição na liberação de somatostatina, resultando em um aumento de $\mathrm{GH}$ (7). Durante o exercício, a secreção de $\mathrm{GH}$ parece ser induzida por ativação de vias alfa-adrenérgicas (adrenalina), enquanto que, no sono (estágios III e IV), o neurotransmissor envolvido seria a serotonina (6).

Bastante relatado na literatura, também, é o efeito estimulante da secreção de $\mathrm{GH}$ induzido pelos aminoácidos. Uma refeição rica em proteínas ou a infusão intravenosa (IV) de uma mistura de aminoá- cidos aumenta o nível plasmático de GH (8). A arginina é o aminoácido estimulante mais potente, no qual sua infusão, ou mesmo administração oral, pode provocar potente estímulo sobre a secreção de GH, efeito que parece decorrer de uma ação inibitória deste aminoácido sobre a liberação de somatostatina $(9,10)$. No entanto, o mecanismo que leva a arginina a estimular a produção de GH ainda não está bem definido (11).

O exercício, especialmente o treinamento de força, também aumenta a concentração sérica de GH (12). Por isso, alguns atletas e praticantes de atividades físicas têmse utilizado da L-arginina com o intuito de obter efeitos ergogênicos, supostamente potencializando a secreção de hormônios anabólicos durante o exercício (13); entretanto, as observações sobre os efeitos metabólicos da suplementação para esta finalidade ainda são controversos. As infusões de outros aminoácidos, incluindo a metionina, fenilalanina, lisina e histidina também promovem aumento relativo de GH na circulação (14), mas nem todos os aminoácidos são efetivos sobre a liberação de GH quando administrados de forma IV ou oralmente.

Merimee e cols., ao administrar IV 183 mg. $\mathrm{kg}^{-1}$ de massa corporal de L-arginina, verificou um aumento significativo sobre a secreção de $\mathrm{GH}$ em mulheres com idades entre 17 e 35 anos (15). Entretanto, essa dosagem não foi capaz de provocar alterações significativas deste hormônio em homens da mesma idade, sendo necessária a dosagem de 367 mg.kg-1 de massa corporal para elevar os níveis de GH. No estudo realizado por Fisker e cols. (11), a infusão IV de $500 \mathrm{mg} \cdot \mathrm{kg}^{-1}$ de massa corporal de L-arginina levou a um aumento significativo na secreção do $\mathrm{GH}$ em indivíduos adultos hígidos. Sob a forma oral, verificou-se que 5 e $9 \mathrm{~g}$ de L-arginina foram capazes de aumentar a secreção de GH em adultos (16). Contudo, no estudo realizado por Isidori e cols. (17), 1,2 g e 2,4 g.dia ${ }^{-1}$ de L-arginina via oral não foram capazes de alterar a secreção do $\mathrm{GH}$ em jovens saudáveis do sexo masculino. Abel e cols. igualmente verificaram que a suplementação de 2,8 e 5,7 g.dia- ${ }^{-1}$ de L-arginina durante um período de 28 dias não modificou secreção do hormônio referido (18).

Baseado nas divergências encontradas na literatura, este trabalho tem como objetivo verificar se a suplementação oral de L-arginina aumenta a secreção do hormônio do crescimento e do IGF-l em indivíduos do sexo masculino.

\section{MATERIAL E MÉTODOS}

Participaram do estudo dezessete voluntários do sexo masculino, com média de idade de 23,45 \pm 0,59 anos, massa 
corporal de $75,27 \pm 2,54 \mathrm{~kg}$, estatura de $177,66 \pm 1,81 \mathrm{~cm}$ e IMC de $23,75 \pm 0,43 \mathrm{~kg} / \mathrm{m}^{2}$, não fumantes, fisicamente ativos e que não utilizavam suplementos vitamínicos e/ou minerálicos. O protocolo foi aprovado pelo Comitê de Ética em Pesquisa do Hospital de Clínicas de Porto Alegre (nº 04/009), e os procedimentos foram conduzidos segundo a resolução específica do Conselho Nacional de Saúde (n ${ }^{\circ}$ 196/96). Todos os indivíduos foram informados detalhadamente sobre os procedimentos utilizados e concordaram em participar de maneira voluntária do estudo, assinando termo de consentimento informado no momento do ingresso.

Foi solicitado aos participantes que, durante o período de avaliações, eles não modificassem sua alimentação e atividades habituais. Para tal verificação, foi solicitado que todos os voluntários preenchessem recordatórios alimentares de três dias durante a semana que antecedia o período de suplementação, bem como durante a semana em que efetivamente era realizada a suplementação. Os recordatórios foram calculados por nutricionista com auxílio do software DietWin Profissional ${ }^{\circledR}$ versão 4.0 para Windows.

Todos os voluntários compareceram em dois momentos diferentes ao Laboratório de Pesquisa do Exercício, no período da manhã e em jejum de 12 horas, separados por um período de 7 dias, para as coletas de sangue e urina. No primeiro dia, após as coletas de sangue e urina, foram entregues aos participantes as cápsulas de suplementação e todos foram devidamente orientados sobre como administrá-las. A suplementação ocorreu por via oral, com a administração de cápsulas orais em um protocolo duplo cego e randomizado. O grupo tratamento recebeu cápsulas de administração oral de cloridrato de L-arginina (SP Farma, lote 103ZA46, Japão), $7 \mathrm{~g}$ ao dia, durante um período de 7 dias. As cápsulas foram administradas em três momentos do dia, ou seja, pela manhã, tarde e noite ( $8 \mathrm{~h}, 16 \mathrm{~h}$ e $24 \mathrm{~h})$. O grupo controle recebeu cápsulas de administração oral de um composto de amido com as mesmas características organolépticas das cápsulas do grupo tratamento. Após o período de suplementação, os voluntários retornavam ao Laboratório para idênticas coletas de sangue e urina.

O sangue foi coletado através de punção venosa na região antecubital e armazenados em tubos Vaccutainer com gel separador, centrifugados para posterior análise da secreção dos hormônios. A coleta de urina ocorreu de forma isolada, não representando o volume de $24 \mathrm{~h}$. Para dosagem de GH utilizou-se um ensaio de quimioluminescência (IMMULITE hGH ${ }^{\circledR}$ - Diagnostic Products Corporation, Los Angeles, CA, USA), com valores de referência de 0,06 a $5 \mathrm{ng} / \mathrm{mL}$, sensibilidade analítica do ensaio $0,01 \mathrm{ng} / \mathrm{mL}$, coeficiente de variação intra-ensaio $5,3 \%$ e o coeficiente de variação inter-ensaio 5,7\%. Para dosagem de IGF-1, foi usado o ensaio imunorradiométrico (DSL-9400 ACTIVE ${ }^{\circledR}$ - Diagnostic Systems Laboratories, Inc, Webster, TX, USA), sensibilidade analítica do ensaio $0,03 \mathrm{ng} / \mathrm{mL}$, coeficiente de variação inter-ensaio $3,7 \%$ e o intra-ensaio menor que $3,3 \%$. A urina foi armazenada em potes plásticos específicos para análise de uréia, que foi avaliada através do método enzimático UV.

Foi avaliada a distribuição das variáveis através do teste de Shapiro-Wilk, e a análise da homocedasticidade das variâncias através do teste de Levene. Os resultados da avaliação nutricional e dos exames de GH, IGF-1 e excreção de uréia foram comparados nos momentos antes e após a suplementação, utilizando-se de teste $t$ para amostras dependentes. Os resultados foram processados em software SPSS versão 12.0 para Windows e estão expressos em média \pm erro-padrão. O nível de significância aceito foi $\mathrm{p}<0,05$.

\section{RESULTADOS}

Os voluntários não modificaram sua alimentação habitual durante o período de suplementação. A tabela 1 apresenta os resultados dos recordatórios alimentares de ambos os grupos nos momentos antes e durante o período de suplementação.

A suplementação aumentou a concentração de uréia na urina do grupo que recebeu L-arginina (de $2684,1 \pm 475,2 \mathrm{mg} / \mathrm{dl}$ para $2967,2 \pm 409,7 \mathrm{mg} / \mathrm{dl}$, $\mathrm{p}=0,002)$, resultado não encontrado no grupo que realizou a suplementação com placebo (de $2570,8 \pm$ $524,7 \mathrm{mg} / \mathrm{dl}$ para $2430,8 \pm 612,5 \mathrm{mg} / \mathrm{dl}, \mathrm{p}=0,567)$.

Tabela 1. Dados dos recordatórios alimentares.

\begin{tabular}{lcccc}
\hline & \multicolumn{2}{c}{$\begin{array}{c}\text { L-arginina } \\
\text { (n= 10) }\end{array}$} & \multicolumn{2}{c}{$\begin{array}{c}\text { Placebo } \\
\text { (n= 7) }\end{array}$} \\
& Antes & Durante & Antes & Durante \\
Calorias ingeridas (kcal) & $2874,36 \pm 164,97$ & $3047,28 \pm 232,71$ & $2945,36 \pm 180,22$ & $2852,72 \pm 219,87$ \\
$\%$ CHO & $56,29 \pm 1,43$ & $53,72 \pm 2,02$ & $54,75 \pm 1,87$ & $56,02 \pm 2,03$ \\
Gramas CHO / kg & $5,23 \pm 0,27$ & $4,98 \pm 0,42$ & $5,02 \pm 0,96$ & $5,13 \pm 2,06$ \\
$\%$ PTN & $16,29 \pm 0,57$ & $16,09 \pm 0,54$ & $17,23 \pm 0,82$ & $16,85 \pm 0,77$ \\
Gramas PTN / kg & $1,49 \pm 0,07$ & $1,39 \pm 0,12$ & $1,54 \pm 0,78$ & $1,50 \pm 0,08$ \\
$\%$ LIP & $27,43 \pm 1,03$ & $29,02 \pm 1,38$ & $29,43 \pm 1,26$ & $27,57 \pm 1,82$ \\
Gramas LIP / kg & $1,12 \pm 0,07$ & $1,39 \pm 0,12$ & $1,27 \pm 0,98$ & $1,22 \pm 1,10$ \\
\hline
\end{tabular}

Resultados dos recordatórios alimentares dos 17 voluntários nos momentos antes e durante o período de suplementação. $\mathrm{CHO}=$ carboidrato; $\mathrm{PTN}=$ proteína; $\mathrm{LIP}=$ lipídeo. . Dados expressos em média \pm ep. 
A figura 1 ilustra os resultados de excreção de uréia nos grupos L-arginina e placebo.

A suplementação não foi capaz de alterar significativamente os valores de $\mathrm{GH}$ em nenhum grupo (de 0,071 $\pm 0,018 \mathrm{ng} / \mathrm{ml}$ para $0,111 \pm 0,034 \mathrm{ng} / \mathrm{ml}$, $\mathrm{p}=0,172$, e de $0,094 \pm 0,022 \mathrm{ng} / \mathrm{ml}$ para $0,062 \pm$ $0,007 \mathrm{ng} / \mathrm{ml}, \mathrm{p}=0,118$, nos grupos L-arginina $\mathrm{e}$ placebo, respectivamente). Assim como os resultados de $\mathrm{GH}$, a suplementação não alterou significativamente os valores de IGF-1 nos grupos (de 420,27 $\pm 36,94 \mathrm{ng} / \mathrm{ml}$ para $418,86 \pm 32,35 \mathrm{ng} / \mathrm{ml}, \mathrm{p}=0,956$, e de $476,48 \pm$ $53,11 \mathrm{ng} / \mathrm{ml}$ para $472,71 \pm 31,50 \mathrm{ng} / \mathrm{ml}, \mathrm{p}=0,924$, nos grupos L-arginina e placebo, respectivamente). As figuras 2 e 3 ilustram os resultados de GH e IGF-1 nos grupos L-arginina e placebo, nos momentos antes e após o período de suplementação.

\section{DISCUSSÃO}

$\mathrm{Na}$ literatura, existe divergência entre os autores sobre a estimulação do GH e IGF-1 pela L-arginina. Este conflito entre os resultados parece ser ocasionado, principalmente, pela forma de administração da Larginina nos voluntários. Neste estudo, avaliamos as respostas de secreção de GH e IGF-1 séricos após a suplementação oral de L-arginina, tendo como parâmetros de controle a alimentação dos voluntários durante o período e a excreção de uréia urinária.

A análise dos recordatórios alimentares dos voluntários foi realizada com o intuito de verificar se os mesmos não alterariam significativamente seu padrão alimentar durante o protocolo de suplementação. Tanto o aporte energético quanto a proteína são importantes na regulação do IGF-l sérico após um período de jejum, e sua medida pode fornecer informações sensíveis a curto prazo no estado nutricional (8). Uma alteração significativa na energia ou proteínas da dieta durante o período de suplementação poderia representar um fator de confusão no desenho deste estudo. Conforme os resultados, os voluntários não modificaram seu padrão alimentar durante o protocolo de estudo, sendo as respostas posteriormente encontradas resultado apenas da suplementação.

Após a suplementação de L-arginina, encontrou-se um aumento significativo da excreção de uréia, demonstrando que a suplementação foi efetiva nesta quantidade. Por ser um intermediário do ciclo da uréia, a suplementação de L-arginina pode provocar um aumento da sua excreção urinária, através do estímulo da enzima arginase, que converte L-arginina em L-ornitina, liberando a uréia (19). Modificações no balanço nitrogenado podem ocasionar paralelamente mudanças no IGF-l sérico (20); entretanto, este achado não foi verificado em nosso estudo.

A administração exógena de arginina ocasiona um aumento significativo da secreção do GH, particularmente quando administrado por via intravenosa (IV). No estudo realizado por Fisker e cols., a infusão IV de $0,5 \mathrm{~g} . \mathrm{kg}^{-1}$ de massa corporal, correspondente a, no máximo, $30 \mathrm{~g}$ de L-arginina, levou a um aumento significativo, porém de curta duração, na secreção do hormônio do crescimento em indivíduos adultos hígidos (11). Da mesma forma, Wideman e cols. (21) também relataram um aumento dos níveis de $\mathrm{GH}$ quando $30 \mathrm{~g}$ de L-arginina foram infundidas IV. Vale ressaltar que a quantidade do aminoácido administrado IV nestes estudos supera em mais de quatro vezes a quantidade ofertada no presente estudo por via oral, dividida ainda em três vezes por dia, totalizando aproximadamente $2,33 \mathrm{~g}$ de L-arginina em cada momento do dia.

Entretanto, encontramos uma divergência nos resultados de estudos que objetivam o efeito da suplementação oral de L-arginina sobre a secreção de GH. Blum e cols. (22) relataram um aumento de $72 \%$ dos níveis de $\mathrm{GH}$ em repouso quando foram administradas $9 \mathrm{~g}$ de L-arginina por dia, durante um período de 4 semanas, bem como o estudo de Isidori e cols. também apontou aumento da secreção de GH após a administração oral de 1,2 g do aminoácido (17). Um estudo bastante recente administrou por via oral diferentes doses de L-arginina $(5,9$ ou $13 \mathrm{~g})$ e placebo em oito indivíduos do sexo masculino, em um modelo randomizado (16). Os autores encontraram um aumento significativo na liberação do $\mathrm{GH}$ nas quantidades de 5 e 9 g por via oral. No entanto, a dosagem de $13 \mathrm{~g}$, além de não aumentar significativamente a secreção de $\mathrm{GH}$, ainda gerou desconfortos gastrointestinais nos voluntários.

Em relação aos resultados de $\mathrm{IGF}-1$, não foram verificadas alterações significativas das suas concentrações após o período de suplementação. A administração oral de L-arginina durante duas semanas aumentou os níveis de IGF-1 em idosos $(23,24)$, entretanto estudos verificando os efeitos em indivíduos jovens ainda são escassos. Chevalley e cols. investigaram o efeito do aumento da concentração plasmática de L-arginina sobre a expressão de IGF-1 em cultura de células osteoblásticas (25). Os autores verificaram que a L-arginina estimula a produção de IGF-1 e síntese de colágeno pelas células, podendo influenciar a formação óssea pela melhora na produção local de IGF-1. Embora esteja bem estabelecida na literatura a ação sinérgica do GH e do IGF-1, alguns dados 


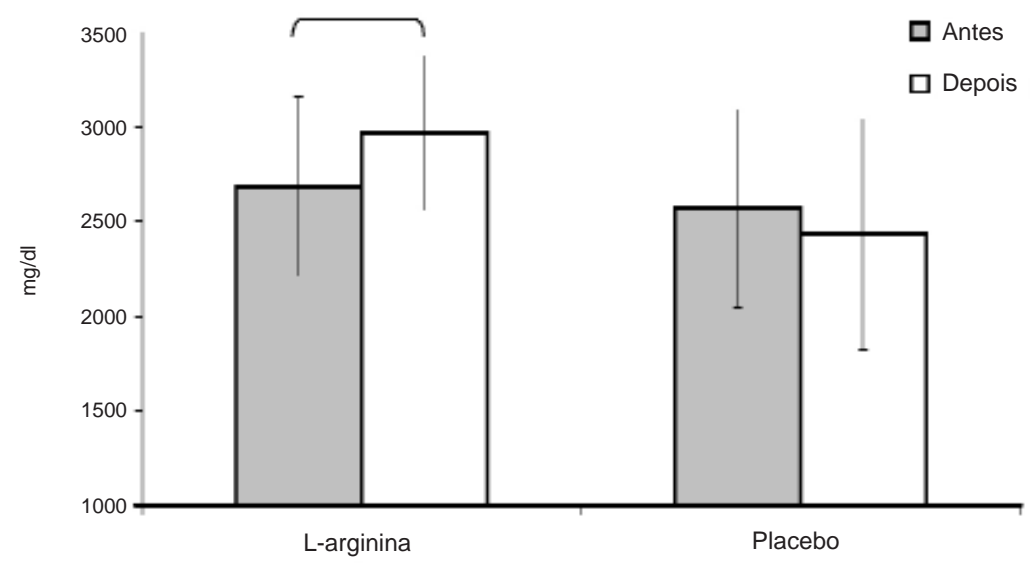

Figura 1. Concentração urinária de uréia antes e após o período de suplementação nos grupos L-arginina $(n=10)$ e placebo $(n=7)$. Dados expressos em média \pm ep. * $\mathrm{p}=0,002$.

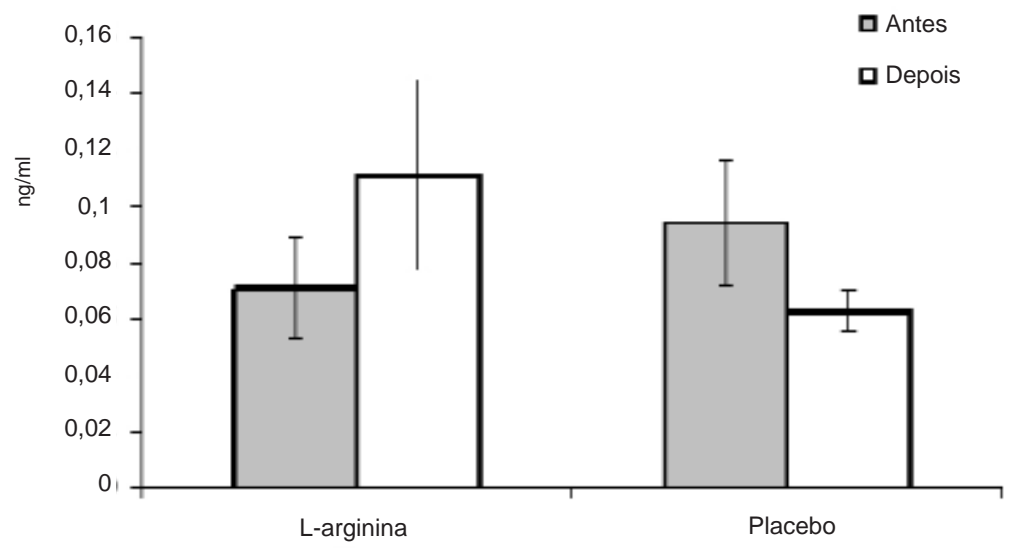

Figura 2. Concentração sérica de $\mathrm{GH}$ antes e após o período de suplementação nos grupos L-arginina $(n=10)$ e placebo $(n=7)$. Dados expressos em média \pm ep.

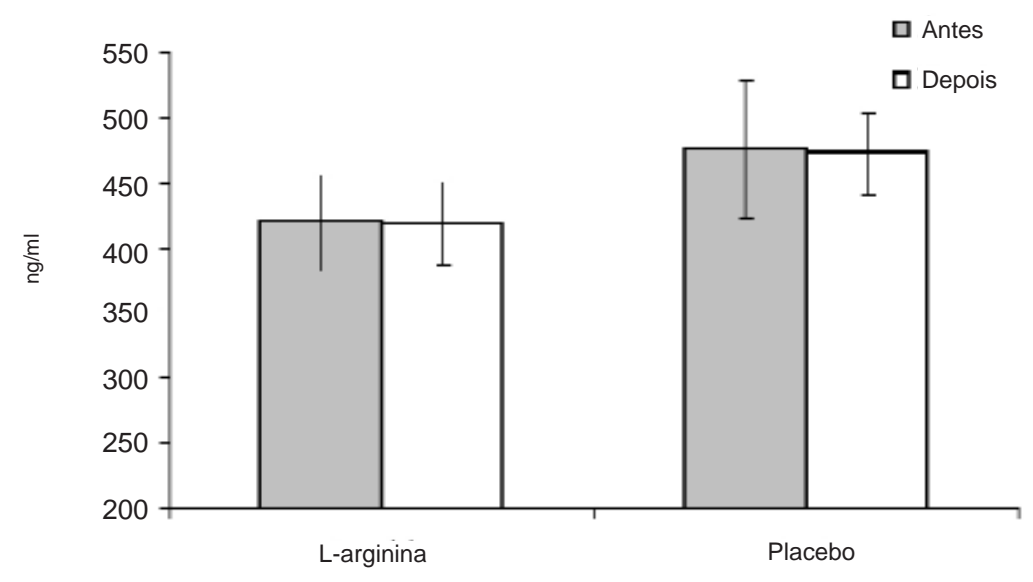

Figura 3. Concentração sérica de IGF-1 antes e após o período de suplementação nos grupos $L$-arginina $(n=10)$ e placebo $(n=7)$. Dados expressos em média \pm ep. 
sugerem que a produção de IGF-1 não é necessariamente dependente dos níveis séricos de GH $(26,27)$, demonstrando uma aparente adaptação deste controle hormonal.

A arginina é conhecida por potencializar a responsividade do GH. Estudos prévios demonstraram um aumento na concentração sérica de GH aproximadamente entre 30 e 60 minutos após a administração exógena de L-arginina, seja ela de forma oral ou IV $(2,16,17,22)$. No presente estudo, a avaliação do GH não foi realizada imediatamente após a administração oral do aminoácido. Portanto, nossos resultados demonstram que, se a L-arginina estimula a secreção do $\mathrm{GH}$, provavelmente o faz em curto prazo, pois este estímulo não foi capaz de permanecer após $8 \mathrm{~h}$ da administração da última dosagem, nem mesmo de permanecer após o uso continuado durante uma semana. Estudos adicionais necessitam ser conduzidos avaliando o tempo de resposta da L-arginina exógena sobre a secreção de hormônios anabólicos.

Em suma, verificamos que a suplementação de L-arginina oral durante o período de uma semana não aumentou a secreção de GH e IGF-1, concluindo, então, que a utilização deste aminoácido é ineficaz para a estimulação destes hormônios.

\section{AGRADECIMENTOS}

Ao FIPE - HCPA e CNPq pelo auxílio financeiro.

\section{REFERÊNCIAS}

1. Mauras N, Haymond MW. Are the metabolic effects of GH and IGF-I separable? Growth Horm IGF Res 2005;15:19-27.

2. Wideman L, Weltman JY, Shah N, Story S, Veldhuis JD, Weltman A. et al. Effects of gender on exercise induced growth hormone release. J Appl Physiol 1999;87:1154-62.

3. Vergani G, Lahr G, Wang ZM, Bartke A, Mayerhofer A. Visualising the expression of a human growth hormone (hGH) transgene in the liver: Intrahepatic regional and intracellular differences of expression are associated with morphological alterations and hepatocellular proliferation. Tissue Cell 1997;29:611-6.

4. Clemmons DR. Role of insulin-like growth factor binding proteins in controlling IGF actions. Mol Cell Endocrinol 1998; $140: 19-24$.

5. Jones JI, Clemmons DR. Insulin-like growth factors and their binding proteins: biological actions. Endocr Rev 1995;16:334.

6. Berne RM, Levy MN, Koeppen BM, Stanton BA. Fisiologia. 5a ed. Rio de Janeiro: Elsevier, 2004.

7. Aires MM. Fisiologia. 2a ed. Rio de Janeiro: GuanabaraKoogan, 1999.

8. Isley WL, Underwood LE, Clemmon DR. Dietary components that regulate serum somatomedin-C concentrations in human. J Clin Invest 1983;71:175-82.

9. Tapiero H, Mathé G, Couvreur P, Tew KD. Dossier: Free amino acids in human health and pathologies. I Arginine. Biomed Pharmacother 2002;56:439-45.
10. Alba-Roth J, Muller OA, Schopohl J, von Werder K. Arginine stimulates growth hormone secretion by suppressing endogenous somatostatin secretion. J Clin Endocrinol Metab 1988;67:1186-9.

11. Fisker S, Nielsen S, Ebdrup L, Bech JN, Christiansen JS, Pedersen $\mathrm{EB}$, et al. L-arginine-induced growth hormone secretion is not influenced by co-infusion of the nitric oxide synthase inhibitor N-monomethyl-L-arginine in healthy men. Growth Horm IGF Res 1999;9:69-73.

12. Chromiak JA, Antonio J. Use of amino acids as growth hormone-releasing agents by athletes. Nutrition 2002;18:657-61.

13. Colombani PC, Bitzi R, Frey-Rindova P, Frey W, Arnold M, Langhans W, et al. Chronic arginine aspartate supplementation in runners reduces total plasma amino acid level at rest and during a marathon run. Eur J Nutr 1999;38:263-70.

14. Knopf RF, Conn JW, Fajans SS, Floyd JC, Guntsche EM, Rull JA. Plasma growth hormone response to intravenous administration of amino acids. J Clin Endocrinol Metab 1965;25:1140-4.

15. Merimee TJ, Rabinowtitz D, Fineberg SE. Arginine-initiated release of human growth hormone. N Engl J Med 1969;280:1434-8.

16. Collier SR, Casey DP, Kanaley JA. Growth hormone responses to varying doses of oral arginine. Growth Horm IGF Res 2005; 15:136-9.

17. Isidori A, Lo Monaco A, Cappa M. A study of growth hormone release in man after oral administration of amino acids. Curr Med Res Opin 1981;7:475-81.

18. Abel T, Knechtle B, Perret C, Eser P, von Arx P, Knecht $\mathrm{H}$. Influence of chronic supplementation of arginine aspartate in endurance athletes on performance and substrate metabolism - a randomized, double-blind, placebo-controlled study. Int J Sports Med 2005;26:344-9.

19. Brody T. Nutritional biochemistry. $2^{\text {nd }}$ ed. London: Academic Press, 1999.

20. Ketelslegers JM, Malter D, Maes M, Underwood LE, Thissen JP. Nutritional regulation of insulin-like growth factor-I. Metabolism 1995;44:50-7.

21. Wideman L, Weltman JY, Patrie JT, Bowers CY, Shah N, Story $\mathrm{S}$, et al. Synergy of L-arginine and growth hormone $(\mathrm{GH})$-releasing peptide-2 on GH release: influence of gender. Am J Physiol Regul Integr Comp Physiol 2000;279:R1467-77.

22. Blum A, Cannon RO 3rd, Costello R, Schenke WH, Csako G. Endocrine and lipid effects of oral L-arginine treatment in healthy postmenopausal women. J Lab Clin Med 2000; $135: 231-7$

23. Kirk SJ, Hurson M, Regan MC, Holt DR, Wasserkrug HL, Barbul A. Arginine stimulates wound healing and imune function in elderly human beings. Surgery 1993;114:155-60.

24. Hurson M, Regan MC, Kirk SJ, Wasserkrug HL, Barbul A. Metabolic effects of arginine in a healthy elderly population. J Parenter Enteral Nutr 1995;19:227-30.

25. Chevalley T, Rizzoli R, Manen D, Caverzasio J, Bonjour JP. Arginine increase insulin-like growth factor-I production and collagen synthesis in osteoblast-like cells. Bone 1998;23:103-9.

26. Gomes RJ, Caetano FH, Hermini HA, Rogatto GP, Luciano E. Efeitos do treinamento físico sobre o hormônio do crescimento $(\mathrm{GH})$ e fator de crescimento semelhante à insulina (IGF1) em ratos diabéticos. Rev Bras Ci Mov 2003;11:55-61.

27. Rubin MR, Kraemer WJ, Maresh CM, Volek JS, Ratamess NA, Vanheest $\mathrm{JL}$, et al. High-affinity growth hormone binding protein and acute heavy resistance exercise. Med Sci Sports Exerc 2005;37:395-403.

\section{Endereço para correspondência:}

Ana Paula Trussardi Fayh

Rua Felizardo 750

90690-200 Porto Alegre, RS

Fax: (51) 3316-5811

E-mail: apfayh@yahoo.com.br 